S. Nishikawa

Nagoya Math. J.

Vol. 95 (1984), 117-124

\title{
ON MAXIMAL SPACELIKE HYPERSURFACES IN A LORENTZIAN MANIFOLD
}

\author{
SEIKI NISHIKAWA*
}

Abstract. We prove a Bernstein-type property for maximal spacelike hypersurfaces in a Lorentzian manifold.

\section{§1. Introduction}

The object of this note is to prove the following

Theorem A. Let $N$ be a Lorentzian manifold satisfying the strong energy condition. Let $M$ be a complete maximal spacelike hypersurface in $N$. Suppose that $N$ is locally symmetric and has nonnegative spacelike sectional curvature. Then $M$ is totally geodesic.

For the terminology in the theorem, see Section 2.

It has been proved by Calabi [2] (for $n \leqq 4$ ) and Cheng-Yau [4] (for all $n$ ) that a complete maximal spacelike hypersurface in the flat Minkowski $(n+1)$-space $L^{n+1}$ is totally geodesic. In particular, the only entire nonparametric maximal spacelike hypersurfaces in $L^{n+1}$ are spacelike hyperplanes. This is remarkable since the Euclidean counterpart, the Bernstein theorem, holds only for $n \leqq 7$ : the entire nonparametric minimal hypersurfaces in the Euclidean space $R^{n+1}, n \leqq 7$, are hyperplanes (cf. [8]).

Theorem A implies, for instance, that a complete maximal spacelike hypersurface in the Einstein static universe is totally geodesic. In the proof of Theorem A, a refinement of a Bernstein-type theorem of ChoquetBruhat $[5,6]$ will be also given.

\section{§2. Definitions}

First we set up our terminology and notation. Let $N=(N, \bar{g})$ be a

*) Partially supported by the Grant-in-aid for Scientific Research No. 57740024 . Received July 13, 1983. 
Lorentzian manifold with Lorentzian metric $\bar{g}$ of signature $(-,+, \cdots,+)$. $N$ has a uniquely defined torsion-free affine connection $\nabla$ compatible with the metric $\bar{g}$. $\quad N$ is said to satisfy the strong energy condition (the timelike convergence condition in Hawking-Ellis [7]) if the Ricci curvature Ric of $N$ is positive semidefinite for all timelike vectors, that is, if $\overline{R i c}(v, v) \geqq 0$ for every timelike vector $v \in T N$ (cf. $[1,6]) . \quad N$ is called locally symmetric if the curvature tensor $\bar{R}$ of $N$ is parallel, that is, $\nabla \bar{R}=0$. We say that $N$ has nonnegative spacelike sectional curvature if the sectional curvature $\bar{K}(u \wedge v)$ of $N$ is nonnegative for every nondegenerate tangent 2-plane spanned by spacelike vectors $u, v \in T N$.

Let $M$ be a hypersurface immersed in $N . \quad M$ is said to be spacelike if the Lorentzian metric $\bar{g}$ of $N$ induces a Riemannian metric $g$ on $M$. For a spacelike $M$ there is naturally defined the second fundamental form (the extrinsic curvature) $S$ of $M . \quad M$ is called maximal spacelike if the mean (extrinsic) curvature $H=\operatorname{Tr} S$, the trace of $S$, of $M$ vanishes identically. $M$ is maximal spacelike if and only if it is extremal under the variations, with compact support through spacelike hypersurfaces, for the induced volume. $M$ is said to be totally geodesic (a moment of time symmetry) if the second fundamental form $S$ vanishes identically.

\section{§3. Local formulas}

Let $M$ be a spacelike hypersurface in a Lorentzian $(n+1)$-manifold $N=(N, \bar{g})$. We choose a local field of Lorentz orthonormal frames $e_{0}$, $e_{1}, \cdots, e_{n}$ in $N$ such that, restricted to $M$, the vectors $e_{1}, \cdots, e_{n}$ are tangent to $M$. Let $\omega_{0}, \omega_{1}, \cdots, \omega_{n}$ be its dual frame field so that the Lorentzian metric $\bar{g}$ can be written as $\bar{g}=-\omega_{0}^{2}+\sum_{i} \omega_{i}^{2} *$ ) Then the connection forms $\omega_{\alpha \beta}$ of $N$ are characterized by the equations

$$
\begin{aligned}
& d \omega_{i}=-\sum_{k} \omega_{i k} \wedge \omega_{k}+\omega_{i 0} \wedge \omega_{0}, \\
& d \omega_{0}=-\sum_{k} \omega_{0 k} \wedge \omega_{k}, \quad \omega_{\alpha \beta}+\omega_{\beta \alpha}=0 .
\end{aligned}
$$

The curvature forms $\bar{\Omega}_{\alpha \beta}$ of $N$ are given by

$$
\begin{aligned}
& \bar{\Omega}_{i j}=d \omega_{i j}+\sum_{k} \omega_{i k} \wedge \omega_{k j}-\omega_{i 0} \wedge \omega_{0 j}, \\
& \bar{\Omega}_{0 i}=d \omega_{0 i}+\sum_{k} \omega_{0 k} \wedge \omega_{k i},
\end{aligned}
$$

*) We shall use the summation convention with Roman indices in the range $1 \leqq$ $i, j, \cdots \leqq n$ and Greek in $0 \leqq \alpha, \beta, \cdots \leqq n$. 
and we have

$$
\bar{\Omega}_{\alpha \beta}=\frac{1}{2} \sum_{\gamma, \hat{o}} \bar{R}_{\alpha \beta \gamma \delta} \omega_{\gamma} \wedge \omega_{\grave{o}},
$$

where $\bar{R}_{\alpha \beta \gamma \delta}$ are components of the curvature tensor $\bar{R}$ of $N$.

We restrict these forms to $M$. Then

$$
\omega_{0}=0 \text {, }
$$

and the induced Riemannian metric $g$ of $M$ is written as $g=\sum_{i} \omega_{i}^{2}$. From formulas (1)-(4), we obtain the structure equations of $M$

$$
\begin{aligned}
& d \omega_{i}=-\sum_{k} \omega_{i k} \wedge \omega_{k}, \quad \omega_{i j}+\omega_{j i}=0, \\
& d \omega_{i j}=-\sum_{k} \omega_{i k} \wedge \omega_{k j}+\omega_{i 0} \wedge \omega_{0 j}+\bar{\Omega}_{i j}, \\
& \Omega_{i j}=d \omega_{i j}+\sum_{k} \omega_{i k} \wedge \omega_{k j}=\frac{1}{2} \sum_{k, \ell} R_{i j k \ell} \omega_{k} \wedge \omega_{\ell},
\end{aligned}
$$

where $\Omega_{i j}$ and $R_{i j k \ell}$ denote the curvature forms and the components of the curvature tensor $R$ of $M$, respectively. We can also write

$$
\omega_{i 0}=\sum_{j} h_{i j} \omega_{j}
$$

where $h_{i j}$ are components of the second fundamental form $S=\sum_{i, j} h_{i j} \omega_{i}$ $\otimes \omega_{j}$ of $M$. Using (6) in (5) then gives the Gauss formula

$$
R_{i j k \ell}=\bar{R}_{i j k \ell}-\left(h_{i k} h_{j \ell}-h_{i \ell} h_{j k}\right) \text {. }
$$

Let $h_{i j k}$ denote the covariant derivative of $h_{i j}$ so that

$$
\sum_{k} h_{i j k} \omega_{k}=d h_{i j}-\sum_{k} h_{k j} \omega_{k i}-\sum_{k} h_{i k} \omega_{k j}
$$

Then, by exterior differentiating (6), we obtain the Coddazi equation

$$
h_{i j k}-h_{i k j}=\bar{R}_{0 i j k} \text {. }
$$

Next, exterior differentiate (8) and define the second covariant derivative of $h_{i j}$ by

$$
\sum_{\ell} h_{i j k \ell} \omega_{\ell}=d h_{i j k}-\sum_{\ell} h_{\ell j k} \omega_{\ell i}-\sum_{\ell} h_{i \ell k} \omega_{\ell j}-\sum_{\ell} h_{i j \ell} \omega_{\ell k} .
$$

Then we obtain the Ricci formula

$$
h_{i j k \ell}-h_{i j \ell k}=\sum_{m} h_{m j} R_{m i k \ell}+\sum_{m} h_{i m} R_{m j k \ell} .
$$

Let us now denote the covariant derivative of $\bar{R}_{\alpha \beta \gamma \delta}$, as a curvature tensor of $N$, by $\bar{R}_{\alpha \beta \gamma \delta \varepsilon \varepsilon}$. Then restricting on $M, \bar{R}_{0 i j k ; \ell}$ is given by 


$$
\bar{R}_{0 i j k ; \ell}=\bar{R}_{0 i j k \ell}-\bar{R}_{0 i 0 k} h_{j \ell}-\bar{R}_{0 i j 0} h_{k \ell}-\sum_{m} \bar{R}_{m i j k} h_{m \ell},
$$

where $\bar{R}_{0 i j k \ell}$ denote the covariant derivative of $\bar{R}_{0 i j k}$ as a tensor on $M$ so that

$$
\sum_{\ell} \bar{R}_{0 i_{j k} \ell} \omega_{\ell}=d \bar{R}_{0 i j h}-\sum_{\zeta} \bar{R}_{0 \ell j k} \omega_{\ell i}-\sum_{\ell} \bar{R}_{0 i \ell k} \omega_{\ell j}-\sum_{\ell} \bar{R}_{0 i j \ell} \omega_{\ell k} .
$$

The Laplacian $\Delta h_{i j}$ of the second fundamental form $h_{i j}$ is defined by

$$
\Delta h_{i j}=\sum_{k} h_{i j k k} .
$$

From (9) we then obtain

$$
\Delta h_{i j}=\sum_{k} h_{k i j k}+\sum_{k} \bar{R}_{0 i j k k},
$$

and from (10)

$$
h_{k i j k}=h_{k i k j}+\sum_{m} h_{m i} R_{m k j k}+\sum_{m} h_{k m} R_{m i j k} .
$$

Replace $h_{k i k j}$ in (13) by $h_{k k i j}+\bar{R}_{0 k i k j}$ (by (9)) and substitute the right hand side of (13) into $h_{k i j k}$ in (12). Then we obtain

$$
\begin{aligned}
\Delta h_{i j}= & \sum_{k}\left(h_{k k i j}+\bar{R}_{0 k i k j}+\bar{R}_{0 i j k k}\right) \\
& +\sum_{k}\left(\sum_{m} h_{m i} R_{m k j k}+\sum_{m} h_{k m} R_{m i j k}\right) .
\end{aligned}
$$

From (7), (11) and (14) we then obtain

$$
\begin{aligned}
\Delta h_{i j}= & \sum_{k} h_{k k i j}+\sum_{k} \bar{R}_{0 k i k ; j}+\sum_{k} \bar{R}_{0 i j k ; k} \\
& +\sum_{k}\left(h_{k k} \bar{R}_{0 i j 0}+h_{i j} \bar{R}_{0 k 0 k}\right) \\
& +\sum_{m, k}\left(h_{m j} \bar{R}_{m k i k}+2 h_{m k} \bar{R}_{m i j k}+h_{m i} \bar{R}_{m k j k}\right) \\
& -\sum_{m, k}\left(h_{m i} h_{m j} h_{k k}+h_{k m} h_{m j} h_{i k}-h_{k m} h_{m k} h_{i j}-h_{m i} h_{m k} h_{k j}\right) .
\end{aligned}
$$

Now we assume that $N$ is locally symmetric, that is, $\bar{R}_{\alpha \beta \gamma \tilde{\sigma} ; \varepsilon}=0$ and that $M$ is maximal in $N$, so that $\sum_{k} h_{k k}=0$. Then, from (15) we obtain

$$
\begin{aligned}
\sum_{i, j} h_{i j} \Delta h_{i j}= & \sum_{i, j, k} h_{i j}^{2} \bar{R}_{0 k 0 k}+\sum_{i, j, k, m} 2\left(h_{i j} h_{m j} \bar{R}_{m k i k}+h_{i j} h_{m k} \bar{R}_{m i j k}\right) \\
& +\left(\sum_{i, j} h_{i j}^{2}\right)^{2} .
\end{aligned}
$$

*) This is the Lorentzian version of the well-known formula established, for example, in [8]. 


\section{§4. Proof of Theorem A}

Theorem $\mathrm{A}$ is an immediate consequence of the following

Theorem B. Let $N=(N, \bar{g})$ be a locally symmetric Lorentzian $(n+1)$ manifold and $M$ be a complete maximal spacelike hypersurface in $N$. Assume that there exist constants $c_{1}, c_{2}$ such that

(i) $\overline{\operatorname{Ric}}(v, v) \geqq c_{1}$ for all timelike vectors $v \in T N$,

(ii) $\bar{K}(u \wedge v) \geqq c_{2}$ for all nondegenerate tangent 2-planes spanned by spacelike vectors $u, v \in T N$, and

(iii) $c_{1}+2 n c_{2} \geqq 0$.

Then $M$ is totally geodesic.

To prove Theorem B, we first note

Lemma 1. Under the assumptions of Theorem B,

$$
\frac{1}{2} \Delta\left(\sum_{i, j} h_{i j}^{2}\right) \geqq\left(\sum_{i, j} h_{i j}^{2}\right)^{2} .
$$

Proof. For any point $p \in M$, we may choose our frame $\left\{e_{1}, \cdots, e_{n}\right\}$ at $p$ so that $h_{i j}=\lambda_{i} \delta_{i j}$. Then, by assumption (ii) of Theorem B, we have at $p$

$$
\begin{aligned}
\sum_{i, j, k, m} 2 & \left.2 h_{i j} h_{m j} \bar{R}_{m k i k}+h_{i j} h_{m k} \bar{R}_{m i j k}\right) \\
& =\sum_{i, k} 2\left(\lambda_{i}^{2} \bar{R}_{i k i k}+\lambda_{i} \lambda_{k} \bar{R}_{k i i k}\right) \\
& =\sum_{i, k}\left(\lambda_{i}-\lambda_{k}\right)^{2} \bar{R}_{i k i k} \geqq c_{2} \sum_{i, k}\left(\lambda_{i}-\lambda_{k}\right)^{2} \\
& =2 c_{2}\left(n \sum_{i} \lambda_{i}^{2}-\left(\sum_{i} \lambda_{i}\right)^{2}\right)=2 n c_{2} \sum_{i, j} h_{i j}^{2} .
\end{aligned}
$$

Also we have by assumption (i)

$$
\sum_{k} \bar{R}_{0 k 0 k} \geqq c_{1} \text {. }
$$

It then follows from (16) and assumption (iii) that

$$
\begin{aligned}
\frac{1}{2} \Delta\left(\sum_{i, j} h_{i j}^{2}\right) & =\sum_{i, j, k} h_{i j k}^{2}+\sum_{i, j} h_{i j} \Delta h_{i j} \\
& \geqq\left(c_{1}+2 n c_{2}\right)\left(\sum_{i, j} h_{i j}^{2}\right)+\left(\sum_{i, j} h_{i j}^{2}\right)^{2} \\
& \geqq\left(\sum_{i, j} h_{i j}^{2}\right)^{2} .
\end{aligned}
$$

Let $u=\sum_{i, j} h_{i j}^{2}$ be the squared of the length of the second fundamental form of $M$. The proof of Theorem B is complete if we show that $u$ vanishes identically. Recall that from (17), $u$ satisfies 


$$
\Delta u \geqq 2 u^{2} .
$$

Then, by the maximum principle, the result is immediate provided $M$ is compact.

We now assume that $M$ is noncompact and complete. We will modify the maximum principle argument as in [4]. Take a point $p \in M$, and let $r$ denote the geodesic distance on $M$ from $p$ with respect to the induced Riemannian metric. For $a>0$, let $B_{a}(p)=\{x \in M \mid r(x)<a\}$ be the geodesic ball of radius $a$ and center $p$.

Lemma 2. For any $a>0$, there exists a constant $c$ depending only on $n$ such that

$$
u(x) \leqq \frac{c a^{2}\left(1+\left|c_{2}\right|^{1 / 2} a\right)}{\left(a^{2}-r(x)^{2}\right)^{2}}
$$

for all $x \in B_{a}(p)$.

Proof. Assuming that $u$ is not identically zero on $B_{a}(p)$, we consider the function

$$
f(x)=\left(a^{2}-r(x)^{2}\right)^{2} u(x), \quad x \in B_{a}(p) .
$$

Then $f$ attains a nonzero maximum at some point $q \in B_{a}(p)$, for the closure of $B_{a}(p)$ is compact since $M$ is complete. As in [§ 2,3], we may assume that $f$ is $C^{2}$ around $q$. Then we have

$$
\nabla f(q)=0, \quad \Delta f(q) \leqq 0 .
$$

Hence at $q^{*}$

$$
\begin{aligned}
& \frac{\nabla u}{u}=\frac{4 r \nabla r}{a^{2}-r^{2}}, \\
& \frac{\Delta u}{u} \leqq \frac{|\nabla u|^{2}}{u^{2}}+\frac{8 r^{2}}{\left(a^{2}-r^{2}\right)^{2}}+\frac{4(1+r \Delta r)}{a^{2}-r^{2}},
\end{aligned}
$$

from which we obtain

$$
\frac{\Delta u}{u}(q) \leqq \frac{24 r^{2}}{\left(a^{2}-r^{2}\right)^{2}}(q)+\frac{4(1+r \Delta r)}{a^{2}-r^{2}}(q) .
$$

On the other hand, according to [Lemma 1,9], $\Delta r(q)$ is bounded from above by

*) We may concentrate on the case of $q \neq p$ for the proof become simpler when $q=p$. 


$$
\Delta r(q) \leqq \min _{0 \leqq k \leqq r(q)}\left[\frac{n-1}{r(q)-k}-\frac{1}{(r(q)-k)^{2}} \int_{k}^{r(q)}(t-k)^{2} \operatorname{Ric}(\dot{\sigma}(t), \dot{\sigma}(t)) d t\right]
$$

where $\dot{\sigma}(t)$ is the tangent vector of the minimizing geodesic $\sigma:[0, r(q)] \rightarrow M$ from $p$ to $q$ and Ric denote the Ricci curvature of $M$. Also, from (7) and assumption (ii) of Theorem B, Ric $(\dot{\sigma}(t), \dot{\sigma}(t))$ is bounded from below by

$$
\operatorname{Ric}(\dot{\sigma}(t), \dot{\sigma}(t)) \geqq(n-1) c_{2},
$$

since $M$ is maximal spacelike. From (21) and (22) we then obtain

$$
r \Delta r(q) \leqq(n-1)+2(n-1)\left|c_{2}\right|^{1 / 2} r(q) .
$$

It follows from (20) and (23) that

$$
\left(a^{2}-r(q)^{2}\right)^{2} u^{-1} \Delta u(q) \leqq 24 a^{2}+8 n a^{2}\left(1+\left|c_{2}\right|^{1 / 2} a\right) .
$$

From (18) we then obtain

$$
f(q)=\left(a^{2}-r(q)^{2}\right)^{2} u(q) \leqq c a^{2}\left(1+\left|c_{2}\right|^{1 / 2} a\right),
$$

$c$ being a constant depending only on $n$. Since $q$ is the maximum point of $f$ in $B_{a}(p)$, this implies that

$$
\left(a^{2}-r(x)^{2}\right)^{2} u(x) \leqq c a^{2}\left(1+\left|c_{2}\right|^{1 / 2} a\right)
$$

for all $x \in B_{a}(p)$.

Since $M$ is complete, we may fix $x$ in Inequality (19) and let $a$ tend to infinity. Then we obtain $u(x)=0$ for all $x \in M$. This completes the proof of Theorem B.

Remark. Let $N=L^{k+1} \times S^{n-k}$ be the product Lorentzian manifold of the flat Minkowski $(k+1)$-space $L^{k+1}, 1 \leqq k \leqq n$, and $S^{n-k}$, a Riemannian $(n-k)$-manifold of positive constant curvature. Then $N$ satisfies the assumptions of Theorem A. The Einstein static space $N=\left(R,-d t^{2}\right) \times S^{n}$ also satisfies these assumptions.

The Lorentzian $(n+1)$-manifold $S_{1}^{n+1}$ of constant curvature $c>0$, called the de Sitter space, satisfies the assumptions of Theorem $B$ (with $\left.c_{1}=-c n, c_{2}=c\right)$. Theorem $\mathrm{B}$ then gives a refinement of a theorem of Choquet-Bruhat [Theorem 4.6,6]. 


\section{REFERENCES}

[1] J. K. Beem and P. E. Ehrlich, Global Lorentzian geometry, Marcel Dekker, 1981.

[2] E. Calabi, Examples of Bernstein problems for some nonlinear equations, Proc. Symp. Pure Appl. Math., 15 (1970), 223-230.

[ 3 ] S.-Y. Cheng and S.-T. Yau, Differential equations on Riemannian manifolds and their geometric applications, Comm. Pure Appl. Math., 28 (1975), 333-354.

[4] S.-Y. Cheng and S.-T. Yau, Maximal space-like hypersurfaces in the LorentzMinkowski spaces, Ann. of Math., 104 (1976), 407-419.

[ 5 ] Y. Choquet-Bruhat, Maximal submanifolds and submanifolds of constant extrinsic curvature of a Lorentzian manifold, Ann. Scuola Norm. Sup. Pisa, 3 (1976), 361376.

[6] Y. Choquet-Bruhat, A. E. Fischer and J. E. Marsden, Maximal hypersurfaces and positivity of mass, Proceedings of the Enrico Fermi Summer School of the Italian Physical Society, J. Ehlers (ed.), North-Holland, 1979.

[ 7 ] S. W. Hawking and G. F. Ellis, The large scale structure of space-time, Cambridge University Press, 1973.

[8] R. Schoen, L. Simon and S.-T. Yau, Curvature estimates for minimal hypersurfaces, Acta Math., 134 (1975), 275-288.

[9] S.-T. Yau, Harmonic functions on complete Riemannian manifolds, Comm. Pure Appl. Math., 28 (1975), 201-228.

Department of Mathematics

Faculty of Science

Kyushu University

Fukuoka 812

Japan 